\title{
Dissecção da aorta após transplante cardíaco ortotópico: relato de 2 casos
}

\author{
Noedir A. G. STOLF* ${ }^{*}$ Alfredo I. FIORELLI*, Fernando BACAL * Viviane VEIGA*, \\ Ricardo BERNADIS*, Edimar A. BOCCHI*, Carlos ABREU FILHO* ${ }^{*}$ Patrícia M. CURY*
}

RBCCV 44205-501

Stolf N A G, Fiorelli A I, Bacal F, Veiga V, Bernadis R, Bocchi E A, Abreu Filho C, Cury P M - Dissecção da aorta após transplante cardíaco ortotópico: relato de 2 casos. Rev Bras Cir Cardiovasc 2000; 15(2):179-85.

RESUMO: No período de março de 1985 a setembro de 1999, 214 pacientes foram submetidos a transplante cardíaco em conseqüência de cardiomiopatia refratária ao tratamento farmacológico. Dois (0,9\%) pacientes, com idades de 33 e 49 anos, desenvolveram dissecção da aorta torácica como complicação tardia fatal após o transplante cardíaco ortotópico. Na primeira paciente, com cardiomiopatia idiopática, esta complicação ocorreu no 93ํㅡês de evolução, enquanto que no segundo, com cardiomiopatia isquêmica, a ocorrência foi mais precoce, no $11^{\circ}$ mês. A hipertensão arterial sistêmica e o tabagismo estiveram presentes como fatores de risco em ambos os casos. As manifestações clínicas da dissecção ocorreram de forma aguda e catastrófica que impossibilitaram qualquer atitude cirúrgica e, possivelmente, maior atenção desta rara complicação possa modificar a sua péssima evolução natural.

DESCRITORES: Transplante de coração. Transplante de coração, efeitos adversos. Miocardiopatias. Miocardiopatias, efeitos adversos. Aorta, dissecção, fatores de risco.

\section{INTRODUÇÃO}

A dissecção da aorta é uma doença grave que cursa com alta mortalidade e geralmente está associada à história pregressa de hipertensão arterial ou necrose cística da camada média. A calcificação e a aterosclerose da aorta são considerados importantes fatores de risco no desenvolvimento da dissecção. A incidência desta complicação após operações cardíacas é inferior à $1 \%$ e estima-se que seja responsável por cerca de 3 a $5 \%$ das causas de óbitos imediatos e tardios $(1,2)$. No transplante cardíaco as complicações na aorta ocorrem com menor freqüência, porém de manuseio mais difícil.

A conexão entre a aorta do doador e receptor é um potencial sítio de complicações imediatas e tardias, sofrendo interferência de infecções, desproporções anatômicas, técnica cirúrgica inadequada na anastomose, fatores hemodinâmicos, rejeição ou alterações degenerativas do próprio segmento vascular. Contudo, a melhora da pressão sistólica após o transplante, a hipertensão arterial sistêmica secundária ao uso de imunossupressão entre outros fatores tornam a aorta mais susceptível ao aparecimento de aneurismas ou dissecções ${ }^{(3,4-6)}$. O reconhecimento precoce destas alterações após o transplante é de fundamental importância, pois, é a meIhor atitude para alterar a evolução destas complicações fatais ${ }^{(7)}$.

A Tabela 1 apresenta a experiência encontrada na literatura da ocorrência de 18 casos de dissecção da aorta após transplante cardíaco. Nota-se maior incidência da doença no sexo masculino e que a hipertensão arterial sistêmica, a cardiomiopatia isquêmica e a doença de Marfan representaram

Trabalho realizado no Instituto do Coração do Hospital das Clínicas da Faculdade de Medicina da Universidade de São Paulo. São Paulo, SP, Brasil. Recebido para publicação em setembro de 1999.

*Do Instituto do Coração da Faculdade de Medicina da Universidade de São Paulo. Endereço para correspondência: Noedir A.G. Stolf. Rua João Lourenço 386. São Paulo, SP, Brasil. CEP 04508-030. Tel: (011) 3069-5318. e-mail: stolf@incor.usp.br 
Stolf N A G, Fiorelli A I, Bacal F, Veiga V, Bernadis R, Bocchi E A, Abreu Filho C, Cury P M - Dissecção da aorta após transplante cardíaco ortotópico: relato de 2 casos. Rev Bras Cir Cardiovasc 2000; 15(2): 179-85.

importantes fatores de risco. Os pacientes portadores da doença de Marfan que tiveram dissecção da aorta apresentavam faixa etária inferior em relação àqueles que eram portadores de cardiomiopatia isquêmica. A dor torácica súbita, insuficiência cardíaca, períodos de hipotensão arterial e o alargamento de mediastino estiveram presentes em praticamente todos os casos.

Não obstante a incidência de dissecções nos transplantes ortotópicos ter sido maior que nos heterotópicos com cifras de $83,3 \%$ e $16,7 \%$, respectivamente, deve-se considerar que esta última modalidade tem sido realizada com menor freqüência e em situações especiais. A aorta nativa foi sede de eleição das dissecções, ocorrendo em $94,4 \%$ dos casos e com comprometimento praticamente semelhante das regiões ascendente e descendente. Houve um único paciente que apresentou dissecção tardia no segmento aórtico do doador, porém o exame histopatológico do referido segmento revelou sinais sugestivos de doença de Marfan, em doador que foi inicialmente suposto como normal (8).

Nos portadores da doença de Marfan ou nos pacientes onde a dissecção da aorta manifestou-se precocemente, houve maior possibilidade de correção cirúrgica (75\%), com menor mortalidade (25\%); possivelmente este subgrupo de risco tenha recebido maior atenção pós-operatória e também permitiu diagnóstico precoce $(9,10)$. Ao contrário dos pacientes onde a dissecção ocorreu tardiamente, apesar das alterações morfológicas na aorta desenvolverem-se lenta e progressivamente, as manifestações clínicas foram abruptas e, muitas vezes, o diagnóstico foi confirmado somente após a morte. Nestes últimos pacientes a possibilidade de correção cirúrgica foi menor $(46,1 \%)$ e a mortalidade maior $(58,3 \%)$.

Nota-se também, na série de pacientes da Tabela 1 , que metade deles faleceu após a dissecção de aorta e que, em $88,9 \%$ dos casos, o principal fator responsável pelo óbito foi a própria dissecção, caracterizando a gravidade desta doença.

O presente estudo tem por objetivo apresentar a evolução de 2 casos fatais de dissecção de aorta que ocorreram tardiamente ao transplante cardíaco ortotópico, 1 com menos de um ano e o outro após 93 meses de evolução.

TABELA 1

AUTORES E PACIENTES QUE APRESENTARAM DISSECÇÃO DA AORTA APÓS TRANSPLANTE CARDÍACO

\begin{tabular}{|c|c|c|c|c|c|c|c|c|c|c|c|c|}
\hline AUTOR - ANO & $\begin{array}{c}N^{\circ} \\
\text { DO } \\
\text { CASO }\end{array}$ & $\begin{array}{l}N^{\circ} \\
D E \\
T X\end{array}$ & $\%$ & $\begin{array}{l}\text { IDADE } \\
\text { (ANOS) }\end{array}$ & SEXO & HAS & $\begin{array}{c}\text { ETIOLOGIA } \\
D A \\
\text { CMP }\end{array}$ & $\begin{array}{c}\text { LOCAL DA } \\
\text { DISSECÇÃO } \\
\text { NA AORTA }\end{array}$ & $\begin{array}{c}\text { TEMPO DE } \\
\text { OCORRÊNCIA } \\
\text { APÓS O TX } \\
\text { (MESES) }\end{array}$ & $\begin{array}{l}\text { CORREÇÃO } \\
\text { CIRÚRGICA }\end{array}$ & $\begin{array}{c}\text { ÓBITO } \\
\text { APÓSA } \\
\text { DISSECÇÃO }\end{array}$ & $\begin{array}{c}\text { DISSECÇÃO } \\
\text { COMO } \\
\text { CAUSA DO } \\
\text { ÓBITO }\end{array}$ \\
\hline Coppola $^{5}-93$ & $\begin{array}{l}1 \\
2\end{array}$ & 250 & 0,8 & $\begin{array}{l}56 \\
54\end{array}$ & $\begin{array}{l}M \\
M\end{array}$ & $\begin{array}{l}S \\
S\end{array}$ & $\begin{array}{l}\text { Isquêmica } \\
\text { Isquêmica }\end{array}$ & $\begin{array}{l}A \\
A\end{array}$ & $\begin{array}{l}60 \\
<1\end{array}$ & $\begin{array}{l}\mathrm{N} \\
\mathrm{N}\end{array}$ & $\begin{array}{l}S \\
S\end{array}$ & $\begin{array}{l}S \\
S\end{array}$ \\
\hline Martinelli7 - 94 & 1 & - & - & 51 & $\mathrm{M}$ & $S$ & Isquêmica & $A^{*}$ & 29 & S & $\mathrm{N}$ & - \\
\hline Kesler ${ }^{9}$ - 94 & $\begin{array}{l}1 \\
2 \\
3 \\
4\end{array}$ & 8930 & 0,04 & $\begin{array}{l}26 \\
37 \\
28 \\
26\end{array}$ & $\begin{array}{l}F \\
M \\
M \\
M\end{array}$ & $\begin{array}{l}N \\
N \\
N \\
N\end{array}$ & $\begin{array}{l}\text { Marfan } \\
\text { Marfan } \\
\text { Marfan } \\
\text { Marfan }\end{array}$ & $\begin{array}{c}- \\
- \\
D \\
A^{* *}\end{array}$ & $\begin{array}{c}1 \\
1 \\
9 \\
29\end{array}$ & $\begin{array}{l}S \\
S \\
N \\
N\end{array}$ & $\begin{array}{c}S^{* * * *} \\
N \\
S \\
N\end{array}$ & $\begin{array}{l}\mathrm{N} \\
- \\
\mathrm{S} \\
-\end{array}$ \\
\hline Muluk $^{11}$ - 95 & $\begin{array}{l}1 \\
2\end{array}$ & 734 & 0,3 & $\begin{array}{l}59 \\
58\end{array}$ & $\begin{array}{l}M \\
M\end{array}$ & $\begin{array}{l}S \\
S\end{array}$ & $\begin{array}{l}\text { Idiopática } \\
\text { Isquêmica }\end{array}$ & $\begin{array}{l}\mathrm{D} \\
\mathrm{D}\end{array}$ & $\begin{array}{l}12 \\
29\end{array}$ & $\begin{array}{l}\mathrm{N} \\
\mathrm{N}\end{array}$ & $\begin{array}{l}S \\
S\end{array}$ & $\begin{array}{l}S \\
S\end{array}$ \\
\hline $\mathrm{Pak}^{12}$ - 95 & 1 & - & - & 49 & $M$ & $S$ & Isquêmica & A & 48 & $\mathrm{~S}$ & $\mathrm{~N}$ & - \\
\hline Mullen ${ }^{10}$ - 96 & 1 & - & - & 22 & M & $\mathrm{N}$ & Marfan & A-T-D & 1 & S & $\mathrm{N}$ & - \\
\hline Defraigne $^{13}-97$ & 1 & 140 & 0,7 & 71 & $M$ & $\mathrm{~S}$ & Idiopática & $D^{\star *}$ & 14 & $\mathrm{~N}$ & $\mathrm{~N}$ & - \\
\hline Pathi $^{8}-97$ & 1 & - & - & 59 & $M$ & $\mathrm{~N}$ & Isquêmica & $A^{* * *}$ & 48 & $\mathrm{~S}$ & $\mathrm{~N}$ & - \\
\hline Vigano $^{3}-99$ & $\begin{array}{l}1 \\
2 \\
3\end{array}$ & 442 & 0,7 & $\begin{array}{l}45 \\
52 \\
53\end{array}$ & $\begin{array}{l}M \\
M \\
M\end{array}$ & $\begin{array}{l}S \\
S\end{array}$ & $\begin{array}{l}\text { Isquêmica } \\
\text { Isquêmica } \\
\text { Idiopática }\end{array}$ & $\begin{array}{l}D \\
A^{*} \\
A^{*}\end{array}$ & $\begin{array}{l}36 \\
29 \\
91\end{array}$ & $\begin{array}{l}S \\
S \\
S\end{array}$ & $\begin{array}{l}N \\
N \\
S\end{array}$ & $\begin{array}{l}- \\
- \\
\text { S }\end{array}$ \\
\hline Stolf - 99 & $\begin{array}{l}1 \\
2\end{array}$ & 220 & 0,9 & $\begin{array}{l}49 \\
33\end{array}$ & $\begin{array}{l}M \\
F\end{array}$ & $\begin{array}{l}S \\
S\end{array}$ & $\begin{array}{l}\text { Isquêmica } \\
\text { Idiopática }\end{array}$ & $\begin{array}{l}D \\
D\end{array}$ & $\begin{array}{l}11 \\
93\end{array}$ & $\begin{array}{l}\mathrm{N} \\
\mathrm{N}\end{array}$ & $\begin{array}{l}S \\
S\end{array}$ & $\begin{array}{l}S \\
S\end{array}$ \\
\hline TOTAL & 18 & & & $46 \pm 14$ & $\begin{array}{c}M=16 \\
F=2\end{array}$ & $61 \%$ & & $\begin{array}{l}A=8 \\
D=8\end{array}$ & $\begin{array}{c}30 \pm 29 \\
\mathrm{I}=4(22 \%) \\
\mathrm{T}=14(88 \%)\end{array}$ & $50 \%$ & $44 \%$ & $44 \%$ \\
\hline
\end{tabular}

* - Transplante heterotópico; ** - Dissecção em observação; ${ }^{\star \star \star ~-~ D i s s e c c ̧ a ̃ o ~ d a ~ a o r t a ~ d o ~ d o a d o r ~ t i p o ~ M a r f a n ; ~ * * \star * ~-~ O ́ b i t o ~} 13$ meses após a dissecção por rejeição.

HAS - Hipertensão Arterial Sistêmica; CMP - Cardiomiopatia; Tx - Transplante; M - Masculino; F - Feminino; S - Sim; N - Não; A Ascendente; T - Transvesa; D - Descendente; I - Imediato e T - Tardio 
Stolf N A G, Fiorelli A I, Bacal F, Veiga V, Bernadis R, Bocchi E A, Abreu Filho C, Cury P M - Dissecção da aorta após transplante cardíaco ortotópico: relato de 2 casos. Rev Bras Cir Cardiovasc 2000; 15(2): 179-85.

\section{RELATO DOS CASOS}

\section{Caso № 1}

Paciente de 49 anos, branco, do sexo masculino que há quatro anos apresentou infarto agudo do miocárdio e foi submetido a angioplastia percutânea. Progressivamente, houve o aparecimento de dispnéia aos esforços, que culminou com indicação para transplante cardíaco ortotópico. Associadamente, apresentava hipertensão arterial de longa evolução e era fumante há mais de 20 anos. Os exames pré-operatórios confirmaram presença de insuficiência cardíaca de origem isquêmica e ausência de outras alterações mais pronunciadas no restante do sistema vascular.

O paciente apresentou boa recuperação após 0 transplante, retornando regularmente ao ambulatório, para acompanhamento clínico. O regime de imunossupressão foi composto por ciclosporina, azatioprina e corticóides, modulados em função dos episódios de rejeição e do seu estado clínico. A hipertensão arterial agravou-se com a imunossupressão, porém foi mantida sob controle com hipotensores e diuréticos.

No $10^{\circ}$ mês de seguimento em ambulatório, apresentou dor abdominal de forte intensidade e foi constatada hérnia de hiato por deslizamento com gastrite aguda difusa, que melhorou relativamente com tratamento medicamentoso. Um mês após este evento, apresentou piora brusca do estado geral, que se iniciou novamente com dor abdominal, oligúria, aumento nos valores de uréia e creatinina, progredindo rapidamente com quadro clínico compatível de abdome agudo por isquemia mesentérica e o óbito ocorreu em poucas horas.

A necropsia revelou presença de aterosclerose intensa acometendo a aorta em praticamente toda sua extensão, com dissecção crônica e orifício de entrada de $3 \mathrm{~cm}$ de extensão na região transversa e com reentrada acima do tronco celíaco. Presença de hematoma em organização no fundo de saco da dissecção da aorta abdominal, falsa luz com hematoma recente na aorta transversa com constrição da luz verdadeira, trombose da artéria renal esquerda com infarto antigo, preservando apenas um terço do rim esquerdo (Figura 1). A dissecção estendiase até próximo à linha de sutura, preservando a aorta ascendente e óstios das artérias coronárias.

O coração apresentava importante hipertrofia de ventrículo esquerdo e coronariopatia de grau discreto com espessamento da parede das artérias e com pontos de estenose inferior a 30\%. Havia também presença de infarto pulmonar antigo no lobo inferior direito e aneurisma de artéria carótida direita.

O exame histopatológico da aorta revelou áreas de dissecção aguda com presença de hematomas não organizados infiltrando a parede do vaso e com sinais de resposta inflamatória aguda (Figura 2-A), e áreas de dissecção antiga com manifestações crônicas de degeneração, com íntima da artéria espessada exibindo intensas alterações ateroscleróticas, incluindo calcificações e fibrose (Figura 2-B).

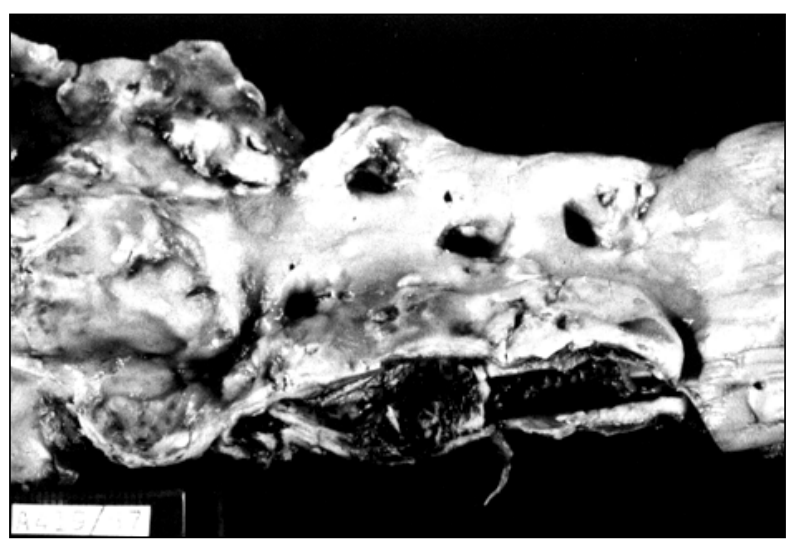

Fig. 1 - Dissecção da aorta torácica até as artérias renais com extenso hematoma na falsa luz.
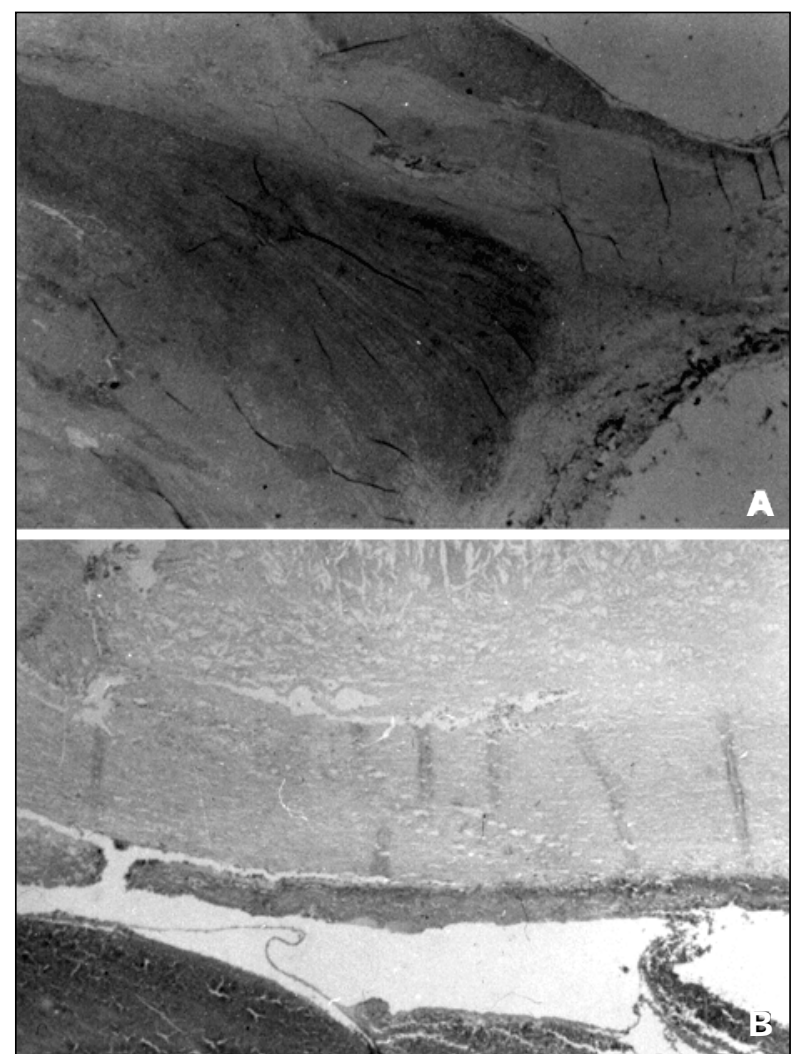

Fig. 2 - A: Aspecto microscópico da dissecção crônica da aorta exibindo trombo em organização na região comprometida. Há trombose recente na superfície da íntima que apresenta espessamento por aterosclerose. B: Aspecto microscópico da dissecção aguda da aorta, podendo-se observar a presença de hematoma recente separando a camada média. 
Stolf N A G, Fiorelli A I, Bacal F, Veiga V, Bernadis R, Bocchi E A, Abreu Filho C, Cury P M - Dissecção da aorta após transplante cardíaco ortotópico: relato de 2 casos. Rev Bras Cir Cardiovasc 2000; 15(2): 179-85.

\section{Caso № 2}

Paciente de 33 anos de idade, do sexo feminino, submetida ao transplante cardíaco ortotópico em virtude de insuficiência cardíaca refratária ao tratamento medicamentoso, em conseqüência de cardiomiopatia pós-parto rapidamente progressiva Foi tabagista durante 20 anos, interrompendo o hábito com o aparecimento da doença cardíaca. No pós-operatório tardio do transplante cardíaco, a paciente desenvolveu hipertensão arterial sistêmica e insuficiência renal, ambas de caráter progressivo e difícil controle. Permaneceu em tratamento dialítico durante um ano, quando, no quinto ano após o transplante cardíaco, foi realizado o transplante renal.

No $93^{\circ}$ mês de seguimento do transplante cardíaco, apresentou, subitamente, dor torácica de forte intensidade, acompanhada de dispnéia de repouso, sudorese e crise hipertensiva. A radiografia de tórax revelou presença de extenso derrame pleural à esquerda e a toracocentese demonstrou que se tratava de hemotórax. A ressonância magnética confirmou a suspeita clínica de dissecção da aorta torácica com início próximo à emergência da artéria subclávia esquerda, estendendo-se até a aorta abdominal. O quadro clínico deteriorou rapidamente, com hipotensão arterial, e a paciente faleceu poucas horas após a confirmação diagnóstica.

\section{COMENTÁRIOS}

A formação de pseudo-aneurismas ou dissecções da aorta após cirurgia cardíaca têm sido referida ocasionalmente, na literatura e geralmente ocorrem em pacientes submetidos a revascularização do miocárdio, que apresentam maior incidência de aterosclerose na aorta com calcificação, após substituição da valva aórtica ou em endocardites $(14,15)$. Embora a ocorrência destas complicações não seja freqüente, o seu reconhecimento é extremamente importante para adoção de terapêutica adequada.

Os pseudo-aneurismas iniciam-se, usualmente, com extravasamento de sangue através de um local da própria linha de sutura ou de maior fragilidade da parede aórtica. Progressivamente, ocorre crescimento do pseudo-aneurisma, atingindo proporções variáveis e a correção cirúrgica está indicada em conseqüência do risco de compressões de estruturas do mediastino ou roturas. Em nossa Instituição, uma criança submetida ao transplante cardíaco ortotópico apresentou, como complicação tardia, a formação de extenso pseudo-aneurisma na linha de sutura entre os cotos aórticos do doador e do receptor. A correção cirúrgica foi indicada e realizada com sucesso (16).
A infecção, principalmente por fungos, exerce papel importante na formação dos pseudo-aneurismas por disseminação hematogênica ou acometimento direto da sutura nas mediastinites $(17,18)$. Em levantamento recente em nossa experiência sobre mediastinite após transplante cardíaco, não se observou qualquer progressão do comprometimento infeccioso para qualquer ponto das diversas suturas realizadas no coração.

A dissecção da aorta como complicação de cirurgia cardiotorácica usualmente ocorre em pontos vulneráveis, como o local de introdução da cânula na aorta, a região de pinçamento aórtico, os orifícios de anastomoses da veia safena ou as linhas de sutura $(1,2,5,14)$. A aterosclerose pode acometer a aorta difusamente e em graus variáveis; contudo, seu papel real como fator desencadeante na dissecção é controverso. Acredita-se que uma placa de ateroma ulcerada pode representar possível ponto de entrada para iniciar o processo de dissecção $(1,2,14)$.

No transplante cardíaco, as doenças da aorta são ainda menos freqüentes e a maioria dos relatos refere-se às aortites, em geral de origem fúngica com formação de pseudo-aneurismas ou roturas $(3,17,18)$.

A dissecção da aorta após o transplante apresenta o mesmo comportamento das dissecções em geral, com desenvolvimento insidioso e manifestação clínica aguda. A maior incidência de dissecções em pacientes com doença de Marfan ou com cardiomiopatia isquêmica é perfeitamente compreensível, pois, na aorta encontram-se alterações anatômicas favoráveis. MULUK et al. (11), em 1995, encontraram aumento significativo de complicações na aorta em transplante de pacientes com cardiomiopatia isquêmica $(2,9 \%)$ em relação aos demais $(0,7 \%)$. Possivelmente, após o transplante a velocidade de propagação aumente pelo ataque imunológico persistente ao endotélio vascular, melhora do padrão hemodinâmico e pelo aparecimento de hipertensão arterial $(5,9,11,19)$. Estas afirmações estão de acordo com observações feitas em outros estudos que demonstram a expansão mais acentuada de aneurismas de aorta abdominal após o transplante cardíaco (20). No nosso segundo relato, o componente da hipertensão arterial severa exerceu papel preponderante no desenvolvimento da dissecção da aorta, considerando-se que se tratava de paciente jovem sem história pregressa de doença aterosclerótica.

$\mathrm{Na}$ presente série, a evolução clínica dos pacientes está de acordo com as descrições já citadas, onde, no paciente com cardiomiopatia isquêmica, a doença aterosclerótica tenha favorecido o aparecimento da dissecção da aorta mais precocemente em relação à paciente com cardiomiopatia pós-parto. A hipertensão arterial sistêmica agravou-se com a 
Stolf N A G, Fiorelli A I, Bacal F, Veiga V, Bernadis R, Bocchi E A, Abreu Filho C, Cury P M - Dissecção da aorta após transplante cardíaco ortotópico: relato de 2 casos. Rev Bras Cir Cardiovasc 2000; 15(2): 179-85.

imunossupressão e contribuiu de forma significativa para acelerar a degeneração da aorta em ambos os casos. Não obstante, o caráter progressivo da doença também pôde ser observado de forma ilustrativa na análise histológica da aorta do paciente com cardiomiopatia isquêmica, onde se encontram áreas de dissecção crônica com infiltrado inflamatório difuso e agudo com hematomas não organizados. COPPOLA et al. (15), em 1993, relataram quadro histológico semelhante ao descrito em paciente também portador de cardiomiopatia isquêmica que morreu por dissecção de aorta após cinco anos de transplante cardíaco ortotópico.

A dissecção da aorta apresenta-se de forma clínica variável; contudo, dor torácica irradiada, sudorese, hipotensão arterial ou síncopes são manifestações presentes com freqüência neste tipo de complicação ${ }^{(14)}$. Em nosso primeiro relato, a ausência de dor torácica possivelmente tenha ocorrido em virtude do componente crônico da dissecção apresentar-se de forma mais exuberante e o quadro agudo ter-se manifestado na aorta toracoabdominal, com compressão do tronco celíaco e artéria renal. Ao contrário do segundo caso, onde a dissecção teve origem próximo à emergência da artéria subclávia esquerda, com rotura para cavidade torácica.

VIGANO et al. (3), em 1999, analisando a incidência de dissecção de aorta após transplante cardíaco, encontraram em apenas $1(0,2 \%)$ paciente, dos 442 realizados de forma ortotópica, e 2 (18,2\%) em 11 pacientes submetidos de forma heterotópica. No transplante heterotópico, a aorta ascendente, próximo ao orifício onde é realizada a anastomose da aorta do doador, em posição terminolateral, sofre estresse adicional resultante das desproporções anatômicas dos vasos e do ângulo de incidência da anastomose. Possivelmente, a adição destes fatores aos que concorrem habitualmente no transplante cardíaco explique a maior incidência de complicações aórticas encontradas neste tipo de modalidade cirúrgica $(3,7)$.

Em geral, a dissecção não acomete a aorta normal do doador, preservando-a até a linha de sutura, a despeito deste segmento vascular sofrer cronicamente agressão imunológica semelhante àquela observada nas artérias coronárias (19). Por outro lado, a aorta do receptor pode apresentar, previamente ao transplante, doença degenerativa em qualquer ponto da sua extensão que se exacerba posteriormente. PATHI et al. (8), em 1997, descreveram a ocorrência de dissecção da aorta ascendente do doador após quatro anos de transplante cardíaco ortotópico, porém o estudo anatomopatológico do segmento comprometido revelou evidências de doença de Marfan, considerado inicialmente como normal. Em um dos nossos pacientes operados na retirada do coração doador, houve dissecção traumática da aorta ascendente durante a infusão de solução cardioplégica, pelo posicionamento inadequado da agulha. A correção da dissecção foi feita com cola biológica e o transplante foi realizado obedecendo aos padrões habituais. O paciente encontra-se vivo e bem há 14 anos.

MARTINELLI et al. (7), em 1995, descreveram o tratamento cirúrgico com sucesso de dissecção da aorta ascendente após 30 meses de transplante heterotópico e MULLEN et al. (10), em 1996, igualmente, descreveram a correção de dissecção recorrente da aorta após transplante cardíaco em portador de síndrome de Marfan. O tratamento cirúrgico da dissecção deve ser realizado, sempre que possível, considerando-se que é a única forma terapêutica capaz de melhorar a sobrevida destes pacientes. A técnica operatória deve obedecer aos mesmos princípios básicos adotados em dissecções de aorta. Contudo, na decisão cirúrgica deve-se ponderar os riscos e benefícios inerentes ao procedimento, ressaltando-se as condições clínicas do paciente e o tipo de dissecção.

As doenças do colágeno, como as síndromes de Marfan e de Ehlers-Danlos são importantes fatores associados à dissecção de aorta, em virtude da degeneração da camada média dos vasos e o depósito de fibras de colágeno, tornando-os mais susceptíveis às dissecções ${ }^{(14)}$. Desta forma, a doença de Marfan oferece risco adicional ao transplante; contudo, isoladamente não se constitui em fator de exclusão. KESLER et al. (9), em 1994, em estudo multicêntrico, encontraram 30 pacientes em lista de espera para transplante cardíaco com doença de Marfan. Desta série, 11 pacientes foram operados e $1(9,1 \%)$ morreu por dissecção de aorta torácica.

Concluindo, a dissecção de aorta é incomum após o transplante cardíaco, porém somente o reconhecimento dos fatores de risco e o diagnóstico precoce podem modificar a evolução maligna que esta doença determina nesta população de pacientes. Deve-se considerar, ainda, que a dor torácica pode estar atenuada após o transplante, o que dificulta o diagnóstico de dissecção, devendo-se sempre valorizar o aparecimento de sopros, insuficiência cardíaca, derrames pleurais, alargamento de mediastino ou dores abdominais sem etiologia aparente. 
Stolf N A G, Fiorelli A I, Bacal F, Veiga V, Bernadis R, Bocchi E A, Abreu Filho C, Cury P M - Dissecção da aorta após transplante cardíaco ortotópico: relato de 2 casos. Rev Bras Cir Cardiovasc 2000; 15(2): 179-85.

RBCCV 44205-501

Stolf N A G, Fiorelli A I, Bacal F, Veiga V, Bernadis R, Bocchi E A, Abreu Filho C, Cury P M - Aortic dissection after orthotopic cardiac transplantation: a report of 2 cases. Rev Bras Cir Cardiovasc 2000; 15(2): 179-85.

ABSTRACT: From March 1985 to September 1999, 214 patients were submitted to cardiac transplantation in consequence of refractory cardiomyopathy to medical therapy. Two patients (0.9\%) aged 33 and 49 years, had developed thoracic aortic dissection as fatal late complication after the orthotopic cardiac transplantation. In the first patient suffering from idiopathic cardiomyopathy this complication occurred in the $93^{\text {rd }}$ month of evolution, while in the second, with ischemic cardiomyopathy, the occurrence was early, in the $11^{\text {th }}$ month. Systemic arterial hypertension and a smoking history had been showed as factors of risk in both cases. The clinical manifestations of the dissection had occurred of acute form and catastrophic that had prevented any surgical management. Greater attention of this rare complication can affect its bad natural evolution.

DESCRIPTORS: Heart transplantation. Heart transplantation, adverse effects. Cardiomyopathies. Cardiomyopathies, adverse effects. Aorta, dissection, risk factors.

\section{REFERÊNCIAS BIBLIOGRÁFICAS}

1 Lam R, Robinson M J, Morales A R - Aortic dissection complicating aortocoronary saphenous vein bypass. Am J Clin Pathol 1977; 68: 729-35.

2 Sabri M N, Henry D, Wechsler A S, DiSciascio G, Vetrovec G W - Late complications involving the ascending aorta after cardiac surgery: recognition and management. Am Heart J 1991; $121(6$ Pt 1): 1779-83.

3 Vigano' $M$, Rinaldi M, D’Armini A M, Pederzolli C, Minzioni G, Grande A M - The spectrum of aortic complications after heart transplantation. Ann Thorac Surg 1999; 68: 105-11.

4 Reichman W, Dyke C, Lee H M, Hanrahan J, Szentpetery S, Sobel M - Symptomatic abdominal aortic aneurysms in long-term survivors of cardiac transplantation. J Vasc Surg 1990; 11: 476-9.

5 Coppola D, Alpern J, Brozena S, McClurken J, Goldman B - Aortic dissection in cardiac allograft recipients: a report of two cases. Arch Pathol Lab Med 1993; 117: 1170-3.

Wada S, Mitsui N, Mukai S et al. - Rupture of donor ascending aorta following heart transplantation. Hiroshima J Med Sci 1994; 43: 73-6.

7 Martinelli L, Rinaldi M, Pederzolli C, Goggi C, Pederzolli $\mathrm{N}$, Vigano $\mathrm{M}$ - Successful treatment of aortic dissection after heterotopic heart transplantation. Ann Thorac Surg 1995; 59: 990-3.

8 Pathi V L, Pillay $T$ M, Wheatley D J, Belcher $P$ R, Naik S K - Marfanoid aneurysm in donor aorta after transplantation. Ann Thorac Surg 1997; 63: 1770-1.

9 Kesler K A, Hanosh J J, O'Donnell J et al. - Heart transplantation in patients with Marfan's syndrome: a survey of attitudes and results. J Heart Lung Transplant 1994; 13: 899-904.

10 Mullen J C, Lemermeyer G, Bentley M J - Recurrent aortic dissection after orthotopic heart transplantation. Ann Thorac Surg 1996; 62: 1830-1.

11 Muluk S C, Steed D L, Makaroun M S et al. - Aortic aneurysm in heart transplant recipients. $J$ Vasc Surg 1995; 22: 689-96.

12 Pak $\mathrm{P} \mathrm{H}$, Gillinov $\mathrm{A} \mathrm{M}$, Hruban $\mathrm{R} \mathrm{H}$ et al. - Aortic dissection in a cardiac allograft recipient: a case report. J Heart Lung Transplant 1995; 14: 1003-5.

13 Defraigne J O, Demoulin J C, Detry O, Bertrand O, Limet $\mathrm{R}$ - Successful management of acute aortic dissection in heart transplant recipient. Acta Chir Belg 1997; 97: 141-4.

14 Roberts W C - Aortic dissection: anatomy, consequences, and causes. Am Heart $J$ 1981; 101: 195-214.

15 Palac R T, Strausbaugh L J, Antonovic R, Floten $\mathrm{H}$ $S$ - An unusual complication of cardiac transplantation: infected aortic pseudoaneurysm. Ann Thorac Surg 1991; 51: 479-81.

16 Jatene M B, Barbero-Marcial M, Azeka E, Atik E, Ebaid $\mathrm{M}$ - Ascending aortic aneurysm after pediatric heart transplantation. Case report repaired with success. Presented at the Third Loma Linda International Conference on Pediatric Heart \& Lung Transplantation, California, 1997.

17 Koyanagi T, Minami K, Tenderich G et al. - Thoracic and cardiovascular interventions after orthotopic heart transplantation. Ann Thorac Surg 1999; 67: 1350-4. 
Stolf N A G, Fiorelli A I, Bacal F, Veiga V, Bernadis R, Bocchi E A, Abreu Filho C, Cury P M - Dissecção da aorta após transplante cardíaco ortotópico: relato de 2 casos. Rev Bras Cir Cardiovasc 2000; 15(2): 179-85.

18 Oaks T E, Pae W E, Pennock J L, Myers J L, Pierce W S - Aortic rupture caused by fungal aortitis: successful management after heart transplantation. $J$ Heart Transplant 1988; 7: 162-4.

19 Mehra M R, Ventura H O, Stapleton D D et al. Allograft aortopathy: an in vivo study of donor aorta involvement in cardiac allograft vasculopathy. Am Heart J 1997; 133: 698-702.

20 Ammori B J, Madan M, Bodenham A R, Gough M J A review of the management of abdominal aortic aneurysms in patients following cardiac transplantation. Eur J Vasc Endovasc Surg 1997; 14: 185-90. 